\title{
HLA Polymorphism in a Sample of Iraqi Acute Lymphoid Leukemia Patients
}

$$
\text { تعدد الأثكال الوراثي لمستضدات خلايا الدم البيض البشرية في عينة عراقية من مرضى }
$$

$$
\begin{aligned}
& \text { Ekhlass N Ali Ali H Ad'hiah* Ahssan SH Al-Demardak } \\
& \text { College of Science/ Al-Mustansiryiah University } \\
& \text { *Tropical-Biological Research Unit/ College of Science/ University of Baghdad }
\end{aligned}
$$

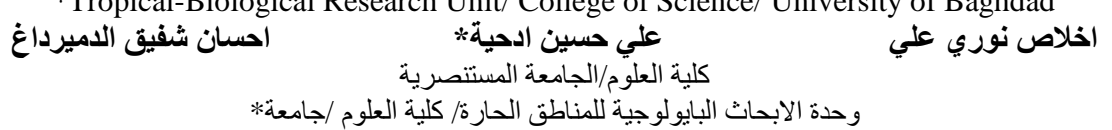

Abstract

The present study aimed to investigate the association between HLA class I (A, B and Cw) and class-II (DR and DQ) antigens and acute lymphoid leukemia (ALL) in Iraqi Arab patients (45 subjects). Based on their clinical subtypes the patients were divided into two subtypes, which were L1 (17 patients)and L2 (28patients). The patients were admitted to Baghdad Teaching Hospital and National Centre of Haematological Diseases at Al-Mustansiryiah University for diagnosis and treatment during the period May 2004 - October 2005. Control sample (blood donors) of 180 subjects, matched with patients for ethnic background (Arab Muslims) was also included. The total ALL patients showed significant increased frequencies of $\mathrm{A2}$ and $\mathrm{B12}$, and a similar observation was made in L1 and L2 subtypes in addition to CW3 The co-occurrence of two antigens belongs to two HLA loci in the same individual was also inspected 14.8,48.1,12.9,9.3, and then their percentage frequencies were determined. Such determination revealed that there were four allelic combinations, which showed a significant increased frequency in the patients. They were A1-DR7, A2-B12, A9-B12, and CW7-DQ1. The RR values of such deviations were 7.65, 26.93, 13.26, and 18.27, respectively of sample Iraqi patients.

Key words: ALL, HLA, Predisposition

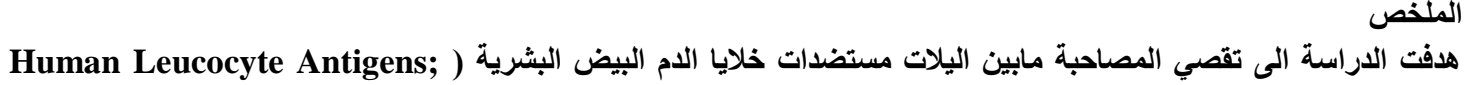

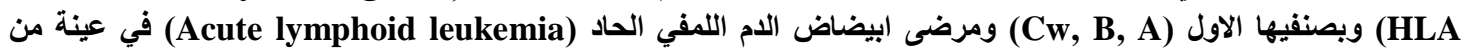

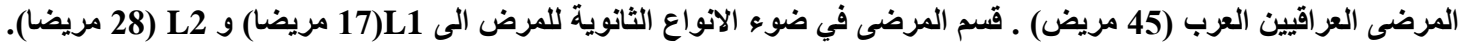

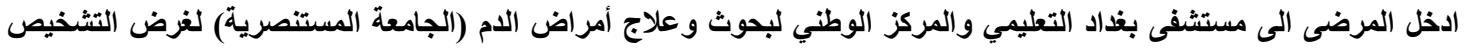

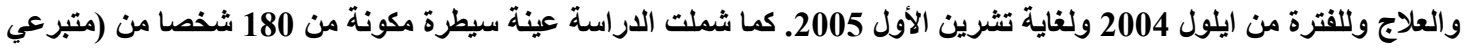

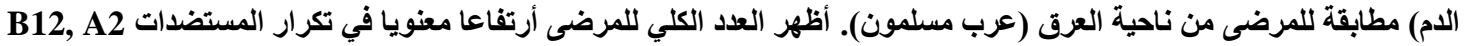

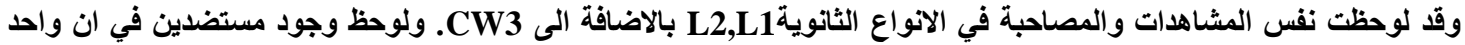

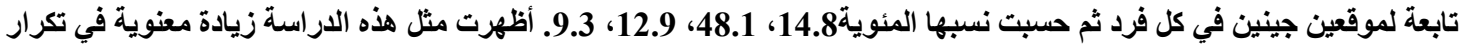

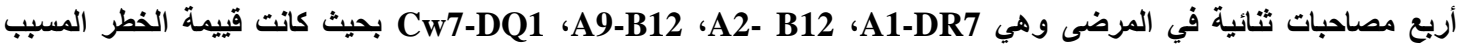

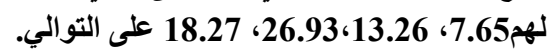

الكلمات المفتاحية: خلايا الدم البيض البشرية، ابيضاض الدم اللمفي الحاد، الاستعداد

\section{Introduction}

Leukemia is a malignant disease of blood-forming organs, characterized by distorted proliferation and development of leukocytes and their precursors in the blood and bone marrow. It is classified according to the degree of cell differentiation as acute or chronic, and according to the predominant type of cell involved as myelogenous or lymphocytic [1]. Acute lymphoid leukemia (ALL) is characterized by an increase in the number of lymphoid cells in bone marrow and an arrest in their maturation, frequently resulting in hematopoietic insufficiency (granulocytopenia, thrombocytopenia or anemia), with or without leukocytosis [2]. In the United States, the annual incidence of ALL is approximately 1,400 deaths a year and it increases in younger (especially those younger than age 50) have a better prognosis than older patients [3], while in Iraq, it was ranked as the first among other 14 types of leukemia in 2005; accounting for $31.85 \%$ of the total histologically verified cases [4].

The a etiology of ALL is not well-defined, although genetic, immunological and environmental factors have been included [3]. However, the nature of these factors is a subject of speculations, but one of the 
genetic factors has gained a special interest because it is able to make an understanding of the a etiological basis of diseases in the ground of genetics and immunity; it is immunogenetic predisposition, which is mainly defined by the major histocompatibility (MHC) system that is known in human as human leukocyte antigen (HLA) system [5]. The extended HLA region on the short arm of chromosome 6 (6p21.3) spans about 7.6 mega base DNA and includes 421 loci, of which 252 are expressed, and is divided into class I, II and III sub regions, and such region contains a number of clustered and duplicated genes, the foremost of which are the HLA class I and class II gene clusters [6]. Class I gene cluster includes the highly polymorphic expressed classical HLA-A, B and C loci, while class II gene cluster includes the polymorphic classical HLA-DR, DQ and DP genes. Both gene clusters encode cell surface heterodimeric proteins that are involved in antigen presentation and $\mathrm{T}$ cell activation [7].

In addition to their role in T cell activation, HLA antigens have demonstrated positive, as well as, negative associations with a wide range of human diseases; autoimmune, viral, bacterial and parasitic diseases are included in such range in addition to malignancies including leukemia [5]. By the beginning of the 1990s, studies on the role of the HLA system in human leukemia susceptibility reached a stage where a large amount of data was generated and many questions were raised but few firm answers were available. However, the general scope of these data agreed that HLA alleles and/or antigens have a role in the a etiology of ALL but such role may depend on type of disease, as well as, ethnic background of patients [8]. Therefore, reliable estimates of phenotype and haplotype frequencies of HLA antigens, alleles, or group of alleles in well-defined ethnic populations are of both theoretical and practical importance. Practical applications of such frequencies include prediction of the probability of finding a suitable HLA-matched donor for stem cell transplantation [9] and the investigation of HLA and disease associations [5]. Accordingly, the present investigation was planned to shed light on the association of HLA polymorphism with ALL in a sample of Iraqi patients. Considerations of the disease subtypes were also made.

Materials and Methods

Forty five ALL patients (21 males and 24 females) were enrolled in the present study. They were admitted to Baghdad Teaching Hospital and National Centre of Hematological Diseases at AlMustansiryiah University for diagnosis and treatment during the period May 2004 - October 2005. Their age (mean \pm standard error) at the time of diagnosis was $26.7 \pm 1.9$ year. For some patients, it was possible to define the subtype (French-American-British classification) of ALL; L1 (17 patients) and L2 (28 patients). The diagnosis of diseases, as well as, AML subtypes was carried out by the consultant medical staff at the clinics and laboratories of the two hospitals. A control sample of 180 apparently healthy individuals (blood donors) was matched with patients for ethnicity (Iraqi Arab Muslims), and they were 88 males and 92 females, and their age was $31.5 \pm 0.9$ year.

\section{Methods}

Venous blood $10 \mathrm{ml}$ was drawn in a heparinized tube, and then it was subjected to a density gradient centrifugation using lymph prep as a separating medium to collect lymphocytes. The collected cells were further separated into $\mathrm{T}$ and $\mathrm{B}$ lymphocytes using the nylon wool method. $\mathrm{T}$ cells were phenotype for HLA-class I antigens (A, B and Cw), while B cells were employed in the phenotyping of HLA-class II antigens (DR and DQ) in the micro lymphocyte to toxicity test [10], using a panel of monoclonal antibodies (Biotest Company, Germany) that were able to recognize 8 HLA-A (A1, A2, A3, A9, A10, A11, A19 and A28), 22 HLA-B (B5, B7, B8, B12, B13, B14, B15, B16, B17, B18, B21, B22, B27, B35, B37, B40, B41, B42, B48, B53, B59 and B73), 7 HLA-Cw (Cw1, Cw2, Cw3, Cw4, Cw5, Cw6 and Cw7), 9 HLA-DR (DR1, DR2, DR3, DR4, DR5, DR6, DR7, DR8 and DR10) and 3 HLA-DQ (DQ1, DQ2 and DQ3) antigens on the surface of the tested lymphocytes. The phenotyping was carried out at the tissue typing laboratory of Al-Karama Teaching Hospital.

Statistical Analysis: The association between HLA alleles and AML was expressed in terms of relative risk (RR), etiological fraction (EF) and preventive fraction (PF). The significance of association (positive or negative) was assessed by Fisher's exact probability, which was multiplied by the number of alleles tested at each locus to obtain the corrected probability (Pc) [10]. Such calculations were carried out using a computer package, which was Programmed for Epidemiologists (PEPI) version 4.0. 


\section{Results}

HLA Antigen Frequencies in Total Patients

Out of 49 antigens tested, 6 antigens showed a significant corrected variation between ALL patients and controls. Antigens A2(68.5 vs. 36.1\%), B12 (66.7vs. 10.6\%), B17 (14.8vs. 2.8\%), showed increased frequencies in patients, while A19 (3.7vs. 33.9\%), DR1 (5.6 vs. 23.9\%) and DR2 (5.6vs. 28.3\%) showed decreased frequencies in patients as compared with controls. Antigens Cw and DQ did not show any significant variations in comparison with controls. The highest RR was recorded for B12 (16.95), followed by B17 (6.09), A2 (4.20), and finally DR1 (0.19). The associated EF values of such positive associations were $0.63,0.12,0.54$, and 0.19 , respectively Table (1).

Table (1): HLA antigens showing significant variation between acute lymphoid leukemia patients and controls.

\begin{tabular}{|c|c|c|c|c|c|c|c|}
\hline \multirow{3}{*}{ HLA Antigen } & \multirow{2}{*}{\multicolumn{2}{|c|}{$\begin{array}{l}\text { ALL Patients } \\
(\text { No. }=45)\end{array}$}} & \multirow{2}{*}{\multicolumn{2}{|c|}{$\begin{array}{c}\text { Controls } \\
(\text { No. }=180)\end{array}$}} & \multicolumn{3}{|c|}{$\mathbf{E F}$} \\
\hline & & & & & $\mathbf{R R}$ & or & $\mathbf{P c} \leq$ \\
\hline & No. & $\%$ & No. & $\%$ & & $\mathbf{P F}$ & \\
\hline A2 & 38 & 70.4 & 65 & 36.1 & 4.20 & 0.54 & 0.001 \\
\hline A19 & 2 & 3.7 & 61 & 33.9 & 0.08 & 0.29 & 0.001 \\
\hline B12 & 36 & 66.7 & 19 & 10.6 & 16.95 & 0.63 & 0.001 \\
\hline B17 & 8 & 14.8 & 5 & 2.8 & 6.09 & 0.12 & 0.04 \\
\hline DR1 & 3 & 5.6 & 43 & 23.9 & 0.19 & 0.19 & 0.01 \\
\hline DR2 & 3 & 5.6 & 51 & 28.3 & 0.15 & 0.24 & 0.001 \\
\hline
\end{tabular}

HLA Antigen Frequencies in subtypes of ALL: With respect to L1 subtype, two antigens showed a significant increased frequency in the patients, which were A2 (76.5 vs. 36.1\%)and B12 (70.6 vs. $10.6 \%)$ while in L2 patients, the variation was to A2 (64.3 vs. 36.1\%), B12 (64.3 vs. 10.6\%) and Cw3 (14.3 vs. 1.7\%) in comparison with controls Table(2).

Table( 2): HLA antigens showing significant variation between subtypes of acute lymphoid leukemia Patients and controls

\begin{tabular}{|c|c|c|c|c|c|c|c|c|}
\hline \multirow[t]{2}{*}{ HLA Antigen } & & \multicolumn{2}{|c|}{ Patients } & \multicolumn{2}{|c|}{$\begin{array}{l}\text { Controls } \\
(\text { No. = 80) }\end{array}$} & \multirow[t]{2}{*}{$\mathbf{R R}$} & \multirow[t]{2}{*}{$\mathbf{E F}$} & \multirow[t]{2}{*}{$\mathbf{P c} \leq$} \\
\hline & & No. & $\%$ & No. & $\%$ & & & \\
\hline \multirow{2}{*}{ L1 Subtype (No. = 17) } & A2 & 13 & 76.5 & 65 & 36.1 & 5.75 & 0.24 & 0.02 \\
\hline & B12 & 12 & 70.6 & 19 & 10.6 & 20.34 & 0.67 & 0.001 \\
\hline \multirow[t]{3}{*}{ L2 Subtype (No. = 28) } & A2 & 18 & 64.3 & 65 & 36.1 & 3.18 & 0.44 & 0.04 \\
\hline & B12 & 18 & 64.3 & 19 & 10.6 & 15.25 & 0.60 & 0.001 \\
\hline & CW3 & 4 & 14.3 & 3 & 1.7 & 9.83 & 0.16 & 0.05 \\
\hline
\end{tabular}

Frequency of HLA-Two-locus Allelic Association: HLA antigens that showed a significant increased frequency in total ALL patients or the disease subtypes were further inspected in patients and controls, but this time through the co-occurrence of two antigens belong to two loci in the same individual, and then their percentage frequencies were determined. Such determination revealed that there were four allelic combinations, which showed a significant increased frequency in the patients. They were A1DR7 (14.8 vs. 2.2\%), A2-B12 (48.1 vs. 3.3\%), A9-B12 (12.9 vs. 1.1\%) and Cw7-DQ1 (9.3 vs. 5.0\%). The RR values of such deviations were $7.65,26.93,13.26$ and 18.27 , respectively, and the corresponding EF values were 0.13, 0.46, 0.12 and 0.09, respectively Table (3).

Table (3): Antigens of two HLA loci showing significant variation between acute lymphoid leukemia patients and controls.

\begin{tabular}{|c|c|c|c|c|c|c|c|}
\hline \multirow[t]{2}{*}{ HLA-Two-Locus Association } & \multicolumn{2}{|c|}{$\begin{array}{l}\text { ALL Patients } \\
(\text { No. }=45)\end{array}$} & \multicolumn{2}{|c|}{$\begin{array}{c}\text { Controls } \\
(\text { No. }=180)\end{array}$} & \multirow[t]{2}{*}{$\mathbf{R R}$} & \multirow[t]{2}{*}{$\mathbf{E F}$} & \multirow[t]{2}{*}{$\mathbf{P c} \leq$} \\
\hline & No. & $\%$ & No. & $\%$ & & & \\
\hline A1-DR7 & 8 & 14.8 & 4 & 2.2 & 7.65 & 0.13 & 0.01 \\
\hline A2-B12 & 26 & 48.1 & 6 & 3.3 & 26.93 & 0.46 & 0.001 \\
\hline A9-B12 & 7 & 12.9 & 2 & 1.1 & 13.26 & 0.12 & 0.001 \\
\hline Cw7-DQ1 & 5 & 9.3 & 9 & $\mathbf{5 . 0}$ & 18.27 & 0.09 & $\mathbf{0 . 0 1}$ \\
\hline
\end{tabular}

\section{Discussion}

To validate the present control sample, the percentage frequency of antigens that showed a significant variation (A2, A19, B12, DR1 and DR2) between ALL patients and controls were compared with their corresponding frequency in a sample of 180 Iraqi Arab blood and potential kidney donors, in which a recent study has examined HLA-A, -B, -DR and -DQ antigens, with the exception of HLA-Cw antigens [11]. Such comparison revealed approximated frequencies (A9: 22.8 vs. 22.0\%; A19: 32.8 vs. 42.0\%; B5: 30.6 vs. 32.0\%; B17: 2.8 vs. 2.0\%; B27: 5.0 vs. 6.0\%; DR3: 19.4 vs. 16.0\%; DR6: 4.4 vs. $3.0 \%$; 
DR7: 19.4 vs. 16.0\%; DQ1: 23.9 vs. 17.0\%), and Pearson's Chi-square analysis demonstrated no significant difference between these frequencies $\left(\mathrm{X}^{2}=5.658\right.$; D.F. $=8$; $\left.\mathrm{P}>0.05\right)$. Accordingly, the observed associations between HLA antigens and ALL can not be due to a chance occurrence, especially if we consider that the obtained Fisher's exact probability was corrected for the number of antigen tested at each locus as suggested by HLA statisticians [12].

The recorded associations in total ALL patients were either positive or negative. The positive associations involved mainly antigens that were clustered in HLA-class I region (A2, B12, B17, and Cw3). The latter antigen may have importance not just in ALL but in other leukemia's, because collective studies carried by the International Bone Marrow Transplant Registry analyzed a large number (total of 1834) of Caucasian patients with ALL, AML, and CML and demonstrated that HLACw3 and -Cw4 are both susceptibility markers for the three major leukemia [13]. In the present study, the RR for Cw3 was 9.83 in subtype L2 EF value was 0.16 , furthermore, Both observations confirm the importance of this allele in the a etiology of ALL, and it can be considered as a predisposing antigen, but as the EF value did not reach 1.0 (maximum predisposition), other factors are required to trigger such a predisposing ground. If we interpret such result in the context of ALL risk factors it is possible to understand such positive association. One of these factors is viral infection, because viruses, particularly RNA retroviruses, have been found to cause many neoplasms in experimental animals, including leukemia [3]. Furthermore, it was also suggested that $\mathrm{Cw} 3$ and other related HLA-class antigens may be considered as markers coding for immune responsiveness to putative leukemia viruses, and in this regard DeVnies et al. [14] found a significant association between $\mathrm{Cw} 3$ and low in vitro responses to vaccine virus. The same investigators suggested that people positive for $\mathrm{Cw} 3$ or $\mathrm{Cw} 4$ may have a $\mathrm{T}$ cell repertoire that is different from individuals who are negative for these two antigens. This may mean that Cw3 and Cw4 antigens are immune response gene products, but this does not answer the question of how these immune response genes confer low responsiveness against a putative leukemia-inducing agent. However, other data imply that individuals who have neither $\mathrm{Cw} 3$ nor $\mathrm{Cw} 4$ are better able to resist leukemia than those who are positive (13). Additional data suggested that Cw3 and Cw4 may be markers for chronic myeloid leukemia (CML) susceptibility in Caucasian and European patients, while in Mexican patients; it has been demonstrated that both antigens have a protective effect on the acquisition of CML [15]. These findings suggest that the development of ALL is apparently associated with HLA phenotypes but they might be specific for each population, and the present HLA-class I profile is in favour of such presentation, because other class I antigens (A2, B12 and Cw3) showed a strong positive association with the present ALL patients, but such observation has not been recorded in other world AML patients, with the exception of American Caucasian AML patients over 11 years of, in whom $\mathrm{Cw} 7$ were increased [16]. Therefore, the race may have impact on HLA association and diseases, and actually HLA antigens themselves show a population-based frequency variation including Iraqis [11].

With respect to HLA-class II antigens DR7 and DQ1 showed significant positive associations with ALL in either total patients or subtypes of the disease. Again, HLA-class II antigens showed variations in different studies. The strongest association in leukemia reported of HLA-DR53 for ALL [17]. Also, in molecular studies examined HLA-DRB loci in ALL, CML and CLL patients, the homozygous genotype for HLA-DRB4*01 (-DR53) had an increased frequency in patients of a Caucasian origin [8]. In contrast Korean AML patients showed associations with DR9 and DR12 [18,19] a positive association was observed with the allele DRB4*01 (the molecular equivalent of the phenotype B57,DR7,Dw1), which was also increased in the present sample of ALL patients [20] .

Such variations were also encountered when the association was considered in term of two-locus allelic occurrence, and in this regard the results revealed four positive associations, especially A1-DR7, A2B12, A9-B12 and Cw7-DQ1, which were associated with a remarkably high significant RR value of $7.65,26.93,13.26$ and 18.27 , respectively. So, is it possible to consider such allelic combination as a predisposing factor for ALL in Iraqi populations? This is not possible and the current finding must be interpreted with caution because the sample size (45 patients) will certainly hamper reaching such conclusion. However, it has been postulated that HLA is not the only factor in a disease predisposition but other players have their impact in their interaction with HLA antigens. Such interaction is different in regard to different ethnic populations in terms of the predisposing HLA antigen and the triggering 
factors that consequence in the stimulation of an immune response that favours a disease induction. This responsiveness will be probably determined by specific alleles that favor an active induction by antigen and pathogen (bacteria and viruses, for example) and confer predisposition [21].

References

1. Craddock, C. (2009). Acute leukaemias. Medicine. 37: 190-194.

2. Pui, C.H., Robison, L.L. and Look, A.T. (2008). Acute lymphoblastic leukaemia. Lancet. 22: 10301034.

3. Trigg, M.E., Sather, H.N., Reaman, G.H.,Tubergen, D.G., Steinherz, P.G and Gaynon, P.S. (2008). Tenyear survival of children with acute lymphoblastic leukemia: a report from the Childrenś Oncology Group. Leuk Lymphoma. 49: 1142-1154.

4. Iraqi Cancer Registry Center Publications. (2008). Iraqi Cancer Registry 2005. Ministry of Health, Iraqi Cancer Board, Baghdad, 2008.

5. Thorsby, E. and Lie, B.A. (2005). HLA associated genetic predisposition to autoimmune diseases: genes involved and possible mechanisms. Transplant Immunology. 14: 175-182.

6. Trowsdale, J. (2005). HLA genomics in the third millennium, Current Opinion in Immunolology. 17: 498-504.

7. Traherne, J. A. (2008). Human MHC architecture and evolution: implications for disease association studies. International Journal of Immunogenetics. 35: 179-192.

8. Dorak, M. T. (2007). MHC and Leukemia. http://www.dorak.info/mhc/mhcleuk.html.

9. Takemoto, S., Port, F.K., Claas, F. H. J. and Duquesnoy, R. J. (2004). HLA matching for kidney transplantation. Human Immunology. 65: 1489-1505.

10. Ad'hiah, A. H. (1990). Immunogenetic Studies in Selected Human Diseases. Ph.D. Thesis, Department of Human Genetics, University of Newcastle upon Tyne, England.

11. Ad'hiah, A. H. (2009). Distribution of HLA polymorphism in a sample of Iraqi Arabs in comparison with three Arab Gulf populations. Iraqi Journal of Science. 50: 120-125.

12. Tiwari, J. L. and Terasaki, P. I. (1985). The data and statistical analysis. In: Tiwari J. L. and Terasaki, P. I., Eds. HLA and Disease Associations, New York: Springer-Verlag. pp. 18-22.

13. Bortin, M. M., D’Amaro, J., Bach, F. H., Rimm, A. A. and van Rood, J. J. (1987). HLA associations with leukemia. Blood. 70: 227-232.

14. De Vries, R.P., Kreeftenberg, H.G., Loggen, H.G. and Van Rood, J.J. (1977). In vitro immune responsiveness to vaccinea virus and HLA. New England Journal of Medicine. 297: 692-696.

15. Rosas-Cabral, A., Irigoyen, L., Alvarado, L., Vela-Ojeda, J., Ayala-Sánchez, M., Tripp-Villanueva, F., Sánchez, E., Gonzalez-Llaven, J. and Gariglio, P. (2003). HLA CW3 and HLA CW4 have a protective effect on acquisition of chronic myeloid leukemia on Mexican patients. Revista de investigacion Clinica. 55: 423-428.

16. Müller, R. Hasmann, H., Grosse-Wilde, U. Vỏgeler, Chen Bei-jun, R. Dopfer, H.D.,Waller, J.M. (1988). Significant association of acute lymphoblastic leukemia with HLA-Cw7. Genetic Epidemiology. 5:453-461.

17. Dorak, M.T.,Tom, L., Helmut, K.G., Darke, K. Ken, I and Burnett, A. K. (1999). Unravelling an HLADR Association in Childhood Acute Lymphoblastic Leukemia. Blood. 94: 694-700.

18. Kim, Y. H., Cho, J. H. and Yang, D. W. (1990). HLA phenotypes of acute myelogenous leukemia in korea. K. J. C. P. 10: 437-445.

19. Fernandes, T. A. R., Fukai, R., Souza, C. A., Lorand-Metze, I., Magna, L. A. and Kraemer, M. H. S. K. (2010). Molecular identification of the HLA-DRB1-DQB1 for diagnosis and follow-up of acute leukemias. Blood Cells, Molecules, and Diseases. 44: 69-73.

20. Leen, M.P. and Gorski, J. (1997). DRB4 promoter polymorphism in DR7 individuals:Correlation with DRB4 pre-mRNA and mRNA levels. Immunogenetics. 45: 371.

21. Wynn, K. K., Fulton, Z., Cooper, L., Silins, S. L., Gras, S., Archbold, J. K., Tynan, F. E., Miles, J. J., McCluskey, J., Burrows, S. R., Rossjohn, J. and Khanna, R. (2008). Impact of clonal competition for peptide-MHC complexes on the CD8+ T-cell repertoire selection in a persistent viral infection. Blood. 111: 4283-4292. 